\title{
Stress Fluctuations in Triaxial Testing of Angular Grains
}

\author{
Aydin OZBAY 1 \\ Ali Firat CABALAR ${ }^{2}$
}

\begin{abstract}
Stress fluctuations caused by stick-slip instabilities are frequently encountered in laboratory shear testings of granular materials. It is not common to observe stick-slips in angular-shaped granular assemblies, although rounded particles are more prone to this type of behaviour. This paper specifically concerns the deviatoric stress fluctuations in the shearing of coarse angular glass granules. A systematic experimental program comprising triaxial compression tests was realized to investigate the effects of particle size, confining pressure, and strain rate on the stick-slip mechanism. Particle size effect was examined by adopting three separate size distributions. In order to understand the influences of testing conditions on the stress fluctuations, the specimens were tested under four different confining pressures and by applying two distinct strain rates. The results showed that both the particle size and confining pressure greatly affected the stress fluctuations whereas the influence of strain rate was unclear.
\end{abstract}

Keywords: Stick-slip, stress fluctuation, coarse angular grains, triaxial compression.

\section{INTRODUCTION}

Unlike many solid materials which exhibit continuum properties, the mechanical response of granular matters depends greatly on the particle characteristics of their constituents. In addition to the gross mass features (e.g., void ratio, relative density), the own properties of the individual particles also affect the behaviour of granular materials. In this respect, influences of the characteristic features of particles such as shape, size, surface roughness, mineralogy, etc. on the global behaviour of the assembly should be well understood to be able to predict the mechanical response of the granular medium. Mechanical behaviour of granular assemblies are also affected by the characteristics of inter-particle load distribution. Actually, the internal force transfer is not homogeneous throughout the granular medium.

\footnotetext{
Note:

- This paper has been received on June 3, 2019 and accepted for publication by the Editorial Board on January 7, 2020.

- Discussions on this paper will be accepted by September 30, 2021.

- https://doi.org/10.18400/tekderg.573637

1 University of Gaziantep, Civil Engineering Department, Gaziantep, Turkey aozbay@gantep.edu.tr - https://orcid.org/0000-0002-4376-8330

2 University of Gaziantep, Civil Engineering Department, Gaziantep, Turkey cabalar@gantep.edu.tr - https://orcid.org/0000-0002-0390-5652
} 
The forces are concentrated around load-bearing contact networks called force chains [1]. There are some strong force chains as well as the weak ones. The alignment of the strong force chains are approximately parallel to the major principal stress direction, whereas the weaker chains are generally diagonal or orthogonal [2]. While the strong force chains dominate the mechanical behaviour of granular medium, the weak force chains usually contribute to the stability of strong ones [3]. In the case of continued loading and insufficient lateral support, the strong force chains may become unstable and be prone to buckling [4]. Domination of the internal forces is shifted between the force chains. As some strong force chains fail as a result of buckling, the load distribution is rearranged among the existing and/or new force chains. At this stage, two types of deformation can be observed within force chain networks depending on the slip mechanism of particles. When the buckling of force chains occurs suddenly, the deformation is defined as stick-slip type. On the contrary, it is said to be a gradual (steady-state) deformation provided that the evolution of force chains is progressive. On the other hand, since the particles are distinct elements and there is no or little cohesion between them, granular materials may exhibit fluid-like behaviour and thus their global stability depends also on the boundary conditions to a large extent. Therefore, the effect of confinement needs to be explored carefully. In brief, the mechanism that governs the response of granular materials under various loading conditions could be defined to have a three-phase structure: (1) particle characteristics, (2) inter-particle force distribution, and (3) boundary conditions. A similar description was made by Sun et al. [3]. They proposed that granular materials are multiscale intrinsically, i.e. microscale (particle size scale), mesoscale (force chains) and macroscale (bulk matter). Actually, both the particle characteristics and the boundary conditions are relatively easy to understand, and also can be controlled. On the other hand, the internal force distribution through the evolution of force chains needs to be observed and analysed thoroughly.

In practice, some assemblages of granular materials such as cohesionless soils are frequently modelled by using the finite element method (FEM) which depends on the continuum mechanical theory. In this method, the granular medium is divided into a finite number of elements (meshes) whose dimensions are obviously different from the actual particle size. On the other hand, the FEM cannot truly model the inter-particle contact mechanics at grainscale and the evolution of force chain networks [3]. Actually, it can only simulate the boundary conditions to a limited extent. Thus, the FEM is far from describing the mechanical behaviour of granular materials. As an alternative, Cundall [5] introduced a numerical solution called as discrete element method (DEM). Although this method was developed firstly for describing the mechanical behaviour of rock blocks, Cundall and Strack [6] extended the technique to the analysis of granular soils. Over decades, the DEM has been a powerful tool to simulate the mechanics of distinct particles due to its compatibility [7-12]. Fortunately, due to the fast processing capabilities of today's computers, model simulations can be performed more effectively. However, those DEM simulations need to be verified by physical experiments. In the literature, there are numerous experimental studies conducted to have a better understanding on the mechanical behaviour of granular materials [1,13-16]. More specifically, the stick-slip phenomenon has also been studied by many researchers [13,17-19]. Actually, the stick-slip phenomenon occurs in granular materials when particles slide, slip with respect to each other. In some cases, sudden releases of stress followed by gradual increase may be observed during the deformation of granular materials [20]. Stick slip behaviour in granular materials has been investigated by other researchers in many 
different disciplines, such as; Thompson and Grest [21], Feder and Feder [22], Demirel and Granick [23], Miller et al., [24], Nasuno et al. [25], Albert et al. [26], Cain et al. [27], Gourdon and Israelachvili [28]. Earlier attempts on the geotechnical engineering were made by Kim [29] and Duchesne [30]. In those studies, some stick-slip type fluctuations in the mechanical response of granular materials were reported. Later on, Adjemian [31] conducted a number of axisymmetric uniaxial compression tests on dry spherical glass beads and also on Huston sand. The stick-slip mechanism was clearly observed in those tests. The results of that study were presented in the paper of Adjemian and Evesque [32]. Stick-slip behaviour of spherical glass beads were also investigated by Alshibli and Roussel [20] and Roussel [33]. They drew some remarkable conclusions on stick-slip load oscillations. In a recent study, Cabalar and Clayton [34] performed undrained triaxial tests on Leighton Buzzard sand and observed instabilities in the stress-strain behaviour in the form of deviatoric stress, axial strain and pore water pressure jumps. Doanh et al. [35] presented the results of another comprehensive testing facility on water-saturated specimens of glass beads. They observed similar fluctuations in deviatoric stress, volumetric strain and excess pore pressure. More recently, Ozbay and Cabalar [36] performed a series of triaxial compression tests on dry spherical glass beads under various loading conditions. They derived some robust conclusions from their experimental investigations.

In the present study, the authors would like to introduce a new experimental work performed on coarse angular granular materials. The paper is mainly focused on stick-slip deformations of dry angular glass granules. A series of triaxial compression tests were realized to investigate the effects of particle size, confining pressure and strain rate on stick-slip behaviour. Three separate particle size distributions were used in the experiments. The specimens of glass granules were tested under four different confining pressures by applying two distinct strain rates.

\section{EXPERIMENTAL WORK}

\subsection{Materials}

Coarse angular glass granules having various size distributions were used in the experimental study. The materials were supplied from the glass recycling company Akcihan, Istanbul, Turkey. The glass granules were arranged according to the supplier's designation. Three groups of materials were included in the tests. Some properties and coefficients are presented in Table 1 and Figure 1.

Table 1 - Some physical properties of the glass granules

\begin{tabular}{|c|c|c|c|c|c|c|c|c|c|c|}
\hline $\begin{array}{c}\text { Material } \\
\text { Name }\end{array}$ & $\begin{array}{c}\text { Specific } \\
\text { Gravity }\end{array}$ & $\begin{array}{c}\mathbf{D}_{\mathbf{1 0}} \\
(\mathbf{m m})\end{array}$ & $\begin{array}{c}\mathbf{D}_{\mathbf{3 0}} \\
\mathbf{( m m )}\end{array}$ & $\begin{array}{c}\mathbf{D}_{\mathbf{5 0}} \\
\mathbf{( m m )}\end{array}$ & $\begin{array}{c}\mathbf{D}_{\mathbf{6 0}} \\
\mathbf{( m m )}\end{array}$ & $\mathbf{C}_{\mathbf{U}}$ & $\mathbf{C}_{\mathbf{C}}$ & $\begin{array}{c}\text { USCS } \\
\text { Class. }\end{array}$ & $\mathbf{e}_{\mathbf{m i n}}$ & $\mathbf{e}_{\mathbf{m a x}}$ \\
\hline GG1 & 2.50 & 0.32 & 0.50 & 0.60 & 0.65 & 2.03 & 1.20 & SP & 0.32 & 0.51 \\
\hline GG2 & 2.50 & 0.76 & 1.04 & 1.26 & 1.36 & 1.79 & 1.05 & SP & 0.56 & 0.71 \\
\hline GG3 & 2.50 & 0.88 & 1.42 & 1.79 & 1.92 & 2.18 & 1.19 & SP & 0.79 & 0.86 \\
\hline
\end{tabular}



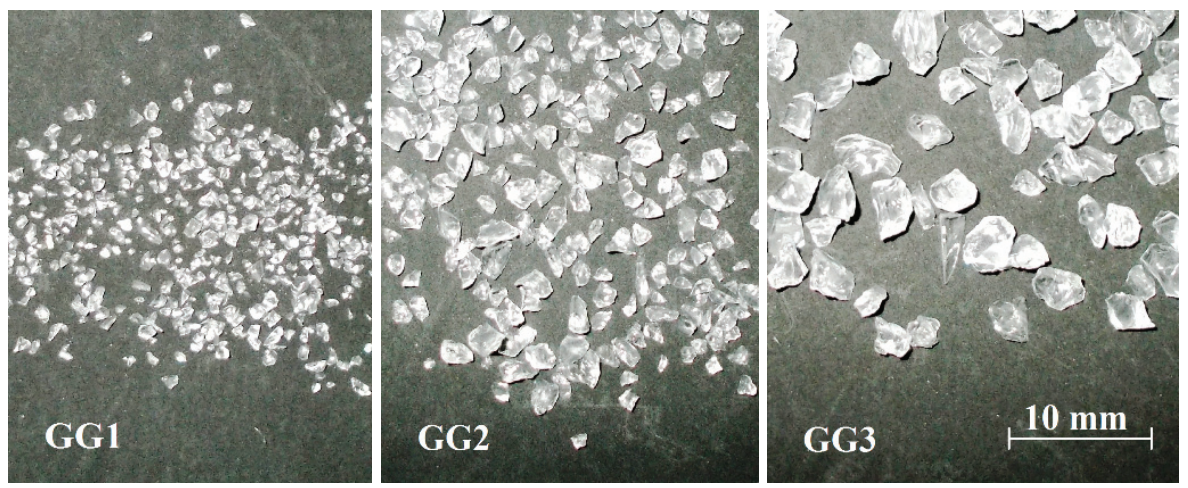

Fig. 1 - Photos of the coarse glass granules

\subsection{Preparation of the Specimens}

The preparation of the glass granule specimens were made by using a cylindrical split mould originally designed for cohesionless soil materials. The angular glass granules were enclosed by $0.3-\mathrm{mm}$ thick latex membranes at periphery and porous stones at the top and at the bottom. The initial diameter and the height of the cylindrical specimens were $38.2 \mathrm{~mm}$ and $77.8 \mathrm{~mm}$, respectively. The specimens were prepared according to the a relative density of about $40 \%$. Dry glass granules within the membrane were sealed by using four O-rings. A small vacuum of nearly $-20 \mathrm{kPa}$ was applied to the inside material in order to hold the glass particles together. The negative pressure was maintained by closing the drainage valves. The split mould was removed gently and a plexiglass pressure chamber was placed around the specimen. Then the chamber was filled with de-aired water and subsequently pressurized to a small confining pressure $(\approx 20 \mathrm{kPa})$. The drainage valves were opened again. The confining pressure was increased to its target value and kept constant during the shearing stage. No saturation was made and the glass granules were left dry throughout the experiments.

\subsection{Testing}

Stick-slip behaviour of the angular glass granules was investigated by using a conventional triaxial loading device. The specimens of glass granules were sheared under axial compression. The axial load and deformation were acquired by means of linear variable differential transformers (LVDTs) connected to a data logger. The drainage valves were open during the tests. Since the glass granule samples were dry, neither volume change nor pore water pressure measurement was done. Thus, in the calculation of deviatoric stress, the correction of the specimen's cross-sectional area was performed by measuring volume change in the cell water.

Twenty four experiments were performed on the angular glass granules (Table 2). In fact, three particle size distributions (labelled as GG1, GG2 and GG3) were utilized to investigate the effect of particle size. Those materials were tested by applying four different confining pressures $(30 \mathrm{kPa}, 50 \mathrm{kPa}, 70 \mathrm{kPa}$ and $100 \mathrm{kPa})$ and two distinct loading rates $(2 \mathrm{~mm} / \mathrm{min}$ and $0.2 \mathrm{~mm} / \mathrm{min}$ ). The glass granule specimens were loaded until an axial strain of about $20 \%$ was reached. 
Table 2 - The details of the tests

\begin{tabular}{|c|c|c|c|c|}
\hline Test No & Test ID & $\begin{array}{c}\text { Material } \\
\text { Type }\end{array}$ & $\begin{array}{c}\text { Confining } \\
\text { Pressure } \\
\end{array}$ & Loading Rate \\
\hline 1 & GG1-30F & GG1 & $30 \mathrm{kPa}$ & $2 \mathrm{~mm} / \mathrm{min}$ \\
\hline 2 & GG1-50F & GG1 & $50 \mathrm{kPa}$ & $2 \mathrm{~mm} / \mathrm{min}$ \\
\hline 3 & GG1-70F & GG1 & $70 \mathrm{kPa}$ & $2 \mathrm{~mm} / \mathrm{min}$ \\
\hline 4 & GG1-100F & GG1 & $100 \mathrm{kPa}$ & $2 \mathrm{~mm} / \mathrm{min}$ \\
\hline 5 & GG1-30S & GG1 & $30 \mathrm{kPa}$ & $0.2 \mathrm{~mm} / \mathrm{min}$ \\
\hline 6 & GG1-50S & GG1 & $50 \mathrm{kPa}$ & $0.2 \mathrm{~mm} / \mathrm{min}$ \\
\hline 7 & GG1-70S & GG1 & $70 \mathrm{kPa}$ & $0.2 \mathrm{~mm} / \mathrm{min}$ \\
\hline 8 & GG1-100S & GG1 & $100 \mathrm{kPa}$ & $0.2 \mathrm{~mm} / \mathrm{min}$ \\
\hline 9 & GG2-30F & GG2 & $30 \mathrm{kPa}$ & $2 \mathrm{~mm} / \mathrm{min}$ \\
\hline 10 & GG2-50F & GG2 & $50 \mathrm{kPa}$ & $2 \mathrm{~mm} / \mathrm{min}$ \\
\hline 11 & GG2-70F & GG2 & $70 \mathrm{kPa}$ & $2 \mathrm{~mm} / \mathrm{min}$ \\
\hline 12 & GG2-100F & GG2 & $100 \mathrm{kPa}$ & $2 \mathrm{~mm} / \mathrm{min}$ \\
\hline 13 & GG2-30S & GG2 & $30 \mathrm{kPa}$ & $0.2 \mathrm{~mm} / \mathrm{min}$ \\
\hline 14 & GG2-50S & GG2 & $50 \mathrm{kPa}$ & $0.2 \mathrm{~mm} / \mathrm{min}$ \\
\hline 15 & GG2-70S & GG2 & $70 \mathrm{kPa}$ & $0.2 \mathrm{~mm} / \mathrm{min}$ \\
\hline 16 & GG2-100S & GG2 & $100 \mathrm{kPa}$ & $0.2 \mathrm{~mm} / \mathrm{min}$ \\
\hline 17 & GG3-30F & GG3 & $30 \mathrm{kPa}$ & $2 \mathrm{~mm} / \mathrm{min}$ \\
\hline 18 & GG3-50F & GG3 & $50 \mathrm{kPa}$ & $2 \mathrm{~mm} / \mathrm{min}$ \\
\hline 19 & GG3-70F & GG3 & $70 \mathrm{kPa}$ & $2 \mathrm{~mm} / \mathrm{min}$ \\
\hline 20 & GG3-100F & GG3 & $100 \mathrm{kPa}$ & $2 \mathrm{~mm} / \mathrm{min}$ \\
\hline 21 & GG3-30S & GG3 & $30 \mathrm{kPa}$ & $0.2 \mathrm{~mm} / \mathrm{min}$ \\
\hline 22 & GG3-50S & GG3 & $50 \mathrm{kPa}$ & $0.2 \mathrm{~mm} / \mathrm{min}$ \\
\hline 23 & GG3-70S & GG3 & $70 \mathrm{kPa}$ & $0.2 \mathrm{~mm} / \mathrm{min}$ \\
\hline 24 & GG3-100S & GG3 & $100 \mathrm{kPa}$ & $0.2 \mathrm{~mm} / \mathrm{min}$ \\
\hline
\end{tabular}

\section{RESULTS AND DISCUSSION}

For the purpose of presenting results in a convenient and compact manner, it was preferred to give the graphical outcomes of the experimental study with respect to two of the input parameters namely particle size and loading rate (Figs. 2-7). The figures also include the photos of the failed samples taken at the end of the tests in order to provide a direct inspection of the test results and comparison with the modes of failure. When taking each photo, the most suitable point of view reflecting the details of specimen failure as much as possible was selected. 
It is clear from the stress-strain plots that there are a number of load (or stress) fluctuations observed during the axial compression of glass granule specimens. Some of these oscillations were caused by stick-slip failures which exhibit a dynamic character but some others are due to the gradual deformation of the samples. It is postulated that the mechanism observed in the specimens tested could be because of two main reasons, which are (i) the specimens' own material properties, and (ii) any compliance in the apparatus. In the light of the study by Gajo [37], it has been seen that the influence of system compliance on collapse of sand samples needs to be taken into account. Gajo [37] revealed that the compliance of the loading apparatus can deeply affect the onset of dynamic instability. One of the reasons of the fluctuations observed could be the stiffness of the apparatus itself. However, the present specifically focuses on the material properties of the specimens tested, since the apparatus used during the testing of specimens was the same apparatus, which was not thought to produce a series of fluctuations in some tests, while producing a pretty smooth curves in others. It is possible to distinguish the stick-slip deformations from the gradual ones especially in the tests of GG1 granules which have the smallest particle size among the tested materials (Figs. 2 and 3). Because of having a dynamic nature, the stick-slips result in sharp decreases in deviatoric stress [1,23-28]. Actually, fluctuation mechanism observed during the tests may be attributed to the structure of the specimens. It is postulated that the mechanism observed in the specimens tested could be because of (i) higher number of contact points, and (ii) number of open fabric structure in the specimens. The higher number of contact points may be due to the higher confining pressure values in the specimens. It seems to be possible that the grains in the specimen may be held in by interlocking asperities in a tighter packing leading to a mechanism having higher internal friction between the soil particles. Hence, from the study by Luding [38], the authors considered that, during the increase in deviatoric stress, the particles in the shear band of the specimens tested under high confining pressure values do not move (steady-state) as easy as those of the specimens tested at low confining pressure values, where the motion of the particles is allowed more easily (fluctuation), because it is assumed that the applied force exceeds easily a critical threshold force, although, the specimens tested under higher confining pressure do not exhibit any fluctuation in deviatoric stress during testing of the specimen. Just after a slip phase, the deviatoric stress increases again but the increment is decelerating as the stress approaches its previous level [5]. The stick-slip instabilities may be attributed to simultaneous buckling of all strong force chains at a section. It refers to concurrent collapse of a row of particles. However, in the steady-state deformations, the failure of strong force chains are compensated by the formation of some other force chains which are previously weak or not existing. In fact, there is a realignment or rearrangement of force chains without any sudden collapse in a gradual (non-stick-slip) deformation. As observed from the experiments given in this study, the amplitudes of the stick-slip failures are always greater than those of the steady-state deformations (see the photos) [7].

When the effect of particle size distribution is considered, similar trends are seen in the overall stress-strain behaviours of the glass granules having different sizes. Besides, it is shown that, as the particle size increases, the amplitudes of stick-slip deformations decrease generally. However, the amplitudes of stress fluctuations corresponding to gradual deformations increase in this case. As for the frequency of stick-slip jumps, it is observed that the number of stick-slips are greater in larger particles. No consistent relation was found to be between the strain ratio and characteristic of the stick-slip behaviour (amplitude, 
frequency) observed in the specimens, although deviatoric stress fluctuations were observed to initiate slightly earlier in the specimens with coarser grains than those in the specimens with finer grains.

As expected, the confining pressure is very effective on the general stress-strain behaviour. Increasing the confining pressure results in higher deviatoric stresses. As a consequence, amplitudes of the stick-slip stress fluctuations increase similarly. However, in some tests, there is no stick-slip failure especially at high confining pressures. For instance, no stick-slip deformation is observed in GG1 glass granules tested under a confining pressure of $100 \mathrm{kPa}$ regardless of the strain rate (Figs. 2 and 3). On the other hand, the confining pressure seems ineffective on the amplitudes of gradual deformations. It is hard to talk about the effect of strain rate on the stress fluctuations since the results are very scattered. However, a limited deduction may be possible for the tests conducted under the confining pressure of $30 \mathrm{kPa}$. In those tests, the number of stick-slip jumps decreases as the strain rate increases.

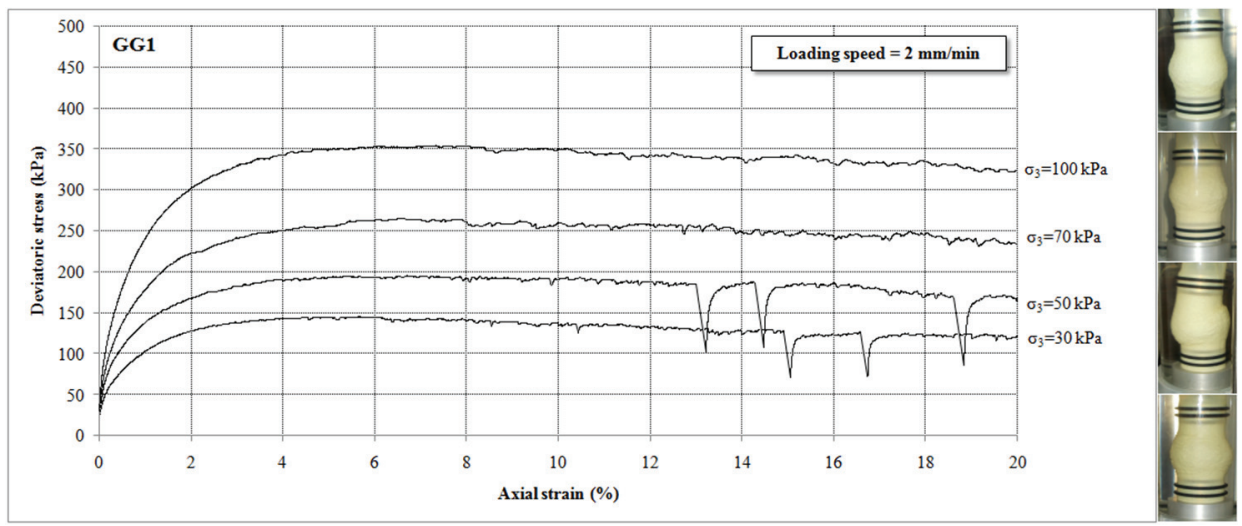

Fig. 2 - GG1 test results for the fast $(2 \mathrm{~mm} / \mathrm{min})$ loading condition

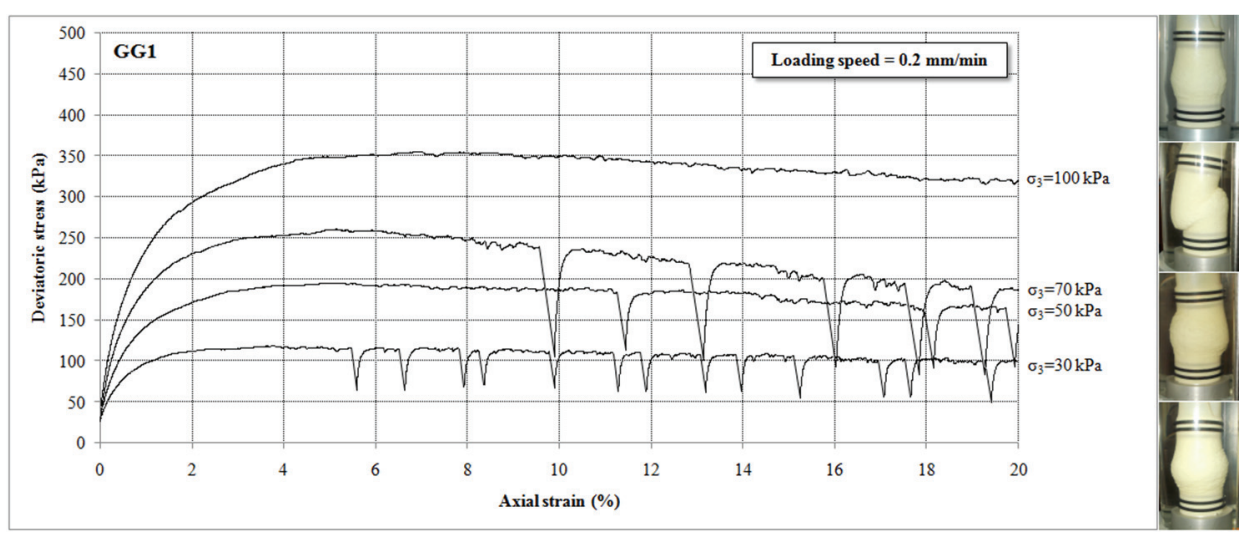

Fig. 3 - GG1 test results for the slow $(0.2 \mathrm{~mm} / \mathrm{min})$ loading condition 


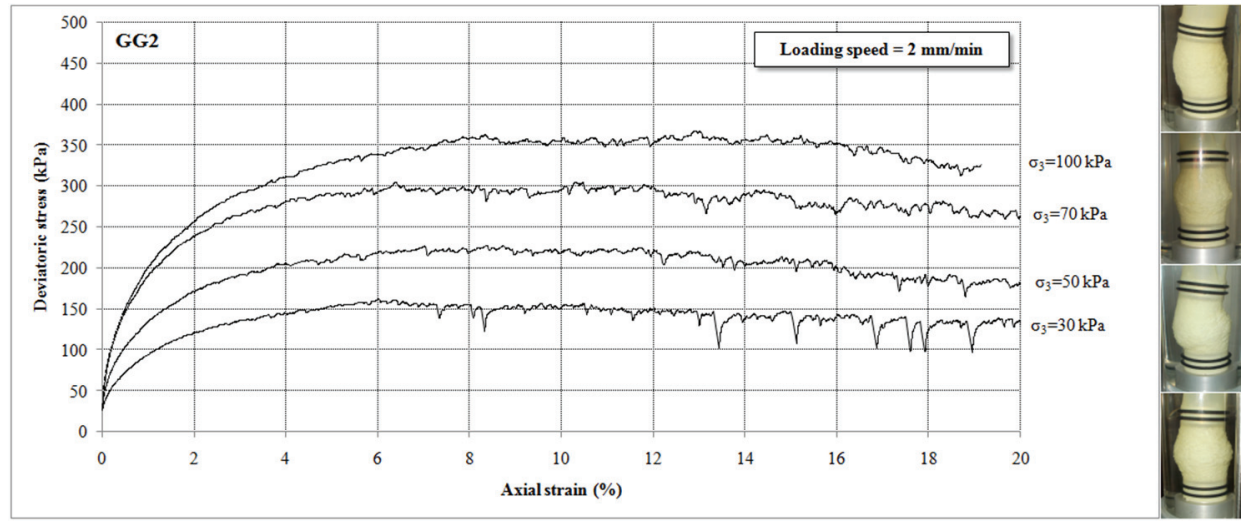

Fig. 4 - GG2 test results for the fast $(2 \mathrm{~mm} / \mathrm{min})$ loading condition

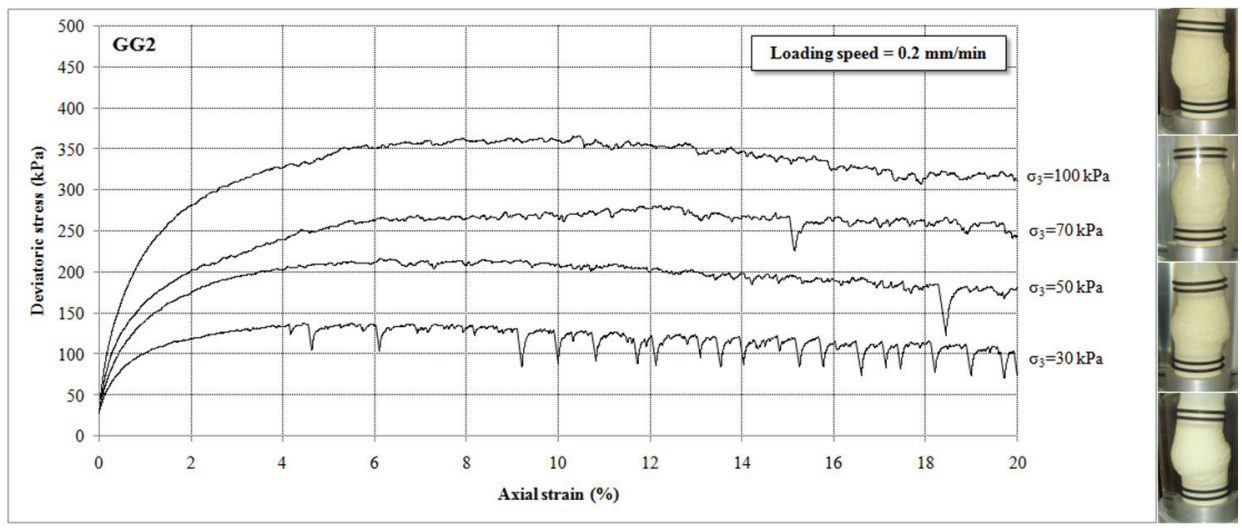

Fig. 5 - GG2 test results for the slow $(0.2 \mathrm{~mm} / \mathrm{min})$ loading condition

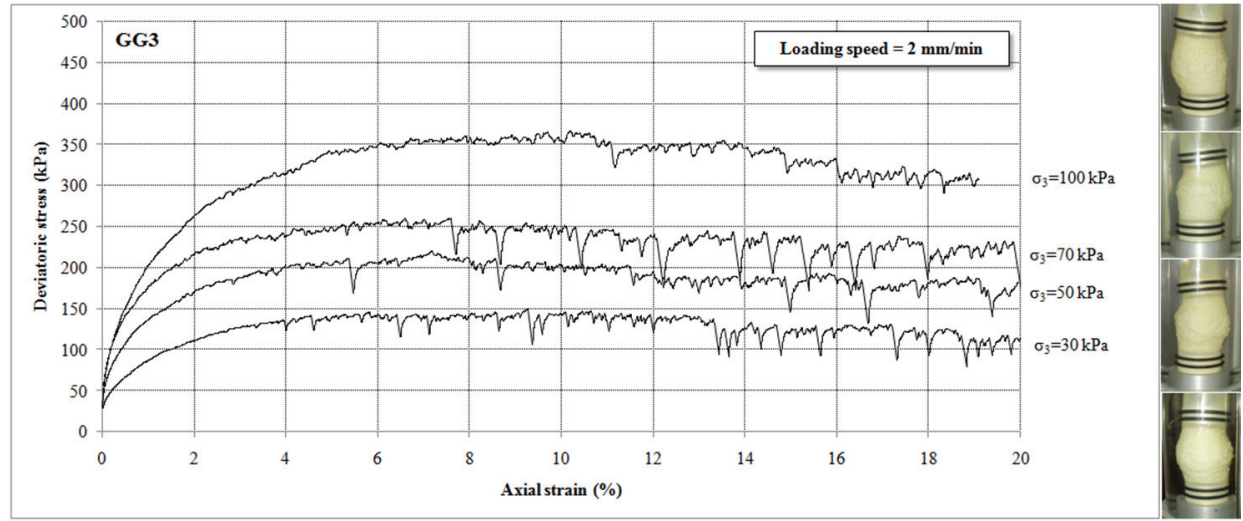

Fig. 6 - GG3 test results for the fast $(2 \mathrm{~mm} / \mathrm{min})$ loading condition 


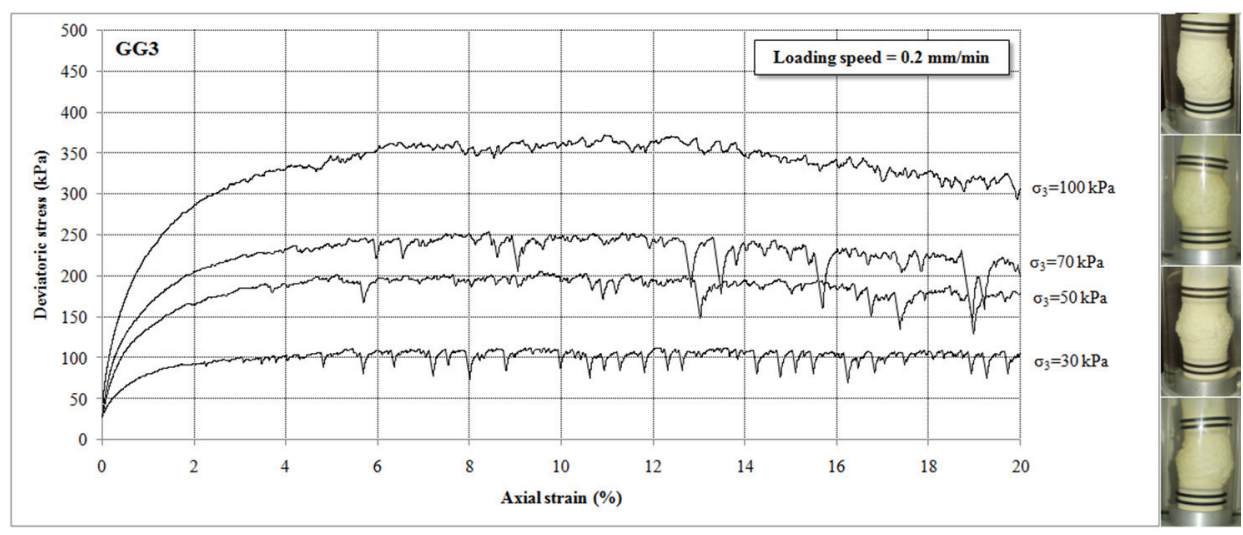

Fig. 7 - GG3 test results for the slow $(0.2 \mathrm{~mm} / \mathrm{min})$ loading condition

Another factor affecting the amplitudes of stick-slip stress fluctuations is the failure mode of the specimens. In some of the tests, the failure planes developed about horizontally (symmetrical bulging). However, there are also some other tests in which the failure planes are diagonal. It is clear from the photos of the failed specimens that greater fluctuation amplitudes are observed when a diagonal shear band formation is apparent. For example, it is very remarkable in the test GG1-70S (i.e., Material: GG1, Confining pressure: $70 \mathrm{kPa}$, Loading speed: $0.2 \mathrm{~mm} / \mathrm{min}$ ) (Fig. 2). This could be explained by the redistribution of stresses on the inclined failure plane [12-17]. Diagonal failure causes a variation in the direction of the shear stress, and the axial normal stress contributes to the shear failure. It implies that, as the inclination angle of the failure plane increases, the extent of the slip in a stick-slip type deformation also increases. This led the authors to consider that type of failure (symmetrical bulging, diagonal plane) was governed by either steady-state or stick-slip deformation during testing the specimens, while yielding might be governed by a different criterion (Mises, Tresca).

\section{CONCLUSIONS}

A number of triaxial compression tests were performed on cylindrical specimens of dry glass granules which contain highly angular particles. The effects of particle size, confining pressure and strain rate on stress fluctuations were investigated. Also failure modes of the specimens were explored in order to relate with the stick-slip amplitudes. The findings of the experimental study are given as follows.

- In all the tests, a number of deviatoric stress fluctuations were observed to some extent. Some of those oscillations were caused by the stick-slip failures and some others by gradual (steady-state) deformations of the glass granule particles. Stress fluctuations corresponding to gradual deformations are existing in all the tests but stick-slip type fluctuations are missing in some cases. The amplitudes of the stick-slip fluctuations in the available tests are greater than those of the gradual fluctuations. 
- $\quad$ Particle size distribution of the granular samples were investigated by using three different sizes. It is found that the amplitudes of stick-slip fluctuations decrease with increasing particle size. However, the fluctuation amplitudes caused by the gradual deformations become greater as the particle size increases. In other words, provided that the stick-slips are ignored, the smaller the particles, the smoother the general stressstrain curve. In addition, finer particles have a less frequency of stick-slip jumps.

- It is also concluded that increasing the confining pressure causes the stick-slip fluctuation amplitudes to increase in most samples. It may be attributed to the variation of deviatoric stress. Besides, there is probably no effect of confining pressure on the steady-state deformation amplitudes.

- Since the results are highly scattered, no such correlation could be established between the strain rate and stress fluctuations.

- The results show that the failure mode of the tested specimens is effective on the stickslip stress oscillations. Although there are horizontal failure planes which are characterized by a symmetric bulging, some of the specimens failed diagonally. Greater stick-slip fluctuation amplitudes were observed in the samples having inclined failure surfaces. It brings about a conclusion that any increase in the inclination angle of the failure plane results in a proportional increase in the value of fluctuation amplitudes.

This suggests that, for the specimen formation in the way employed in the present study, both the particle size and the confining pressure have significant effect on the overall stress-strain behaviour of coarse angular granular materials while the influence of strain rate is unclear.

\section{References}

[1] Cabalar, A.F. Stress fluctuations in granular material response during cyclic direct shear test. Granular Matter, 17(4), 439-446, 2015.

[2] Hanley, K.J., O’Sullivan, C, Wadee, M.A., and Huang, X. Use of elastic stability analysis to explain the stress-dependent nature of soil strength. Royal Society Open Science, 2, 150038, 2015.

[3] Sun, Q., Wang, G., Hu, K. Some open problems in granular matter mechanics. Progress in Natural Science, 19, 523-529, 2009.

[4] Tordesillas, A., Walker, D.M., Lin, Q. Force cycles and force chains. Physical Review $E, 81,011302,2010$.

[5] Cundall, P.A. A computer model for simulating progressive large-scale movements in blocky rock systems. Proc. Symp. Int. Soc. Rock Mech., Nancy, France, 2 (8), 132-150, 1971.

[6] Cundall, P.A., Strack, O.D.L. A discrete numerical model for granular assemblies. Geotechnique, 29 (1), 47-65, 1979.

[7] Antony, S.J., Kuhn, M.R., Barton, D.C., Bland, R. Strength and signature of force networks in axially compacted sphere and non-sphere granular media: micromechanical investigations. J. Phys. D: Appl. Phys. 38, 3944-3952, 2005. 
[8] Salot, C. Gotteland, P., Villard, P. Influence of relative density on granular materials behavior: DEM simulations of triaxial tests. Granular Matter, 11, 221-236, 2009.

[9] Barreto, D., O'Sullivan, C. The influence of inter-particle friction and the intermediate stress ratio on soil response under generalised stress conditions. Granular Matter, 14, 505-521, 2012.

[10] Kozicki, J., Tejchman, J., Mühlhaus, H.-B. Discrete simulations of a triaxial compression test for sand by DEM. Int. J. Numer. Anal. Meth. Geomech., 38, 1923$1952,2014$.

[11] Huang, W.-C., Sung, C.-Y., Liao, H.-Y., Chu, S.-S. Micromechanical behavior of granular materials in direct shear modeling. Journal of the Chinese Institute of Engineers, 38 (4), 469-480, 2015.

[12] Wang, W., Gu, W., Liu, K. Force chain evolution and force characteristics of shearing granular media in Taylor-Couette geometry by DEM. Tribology Transactions, 58, 197206, 2015.

[13] Rathbun, A.P., Marone, C. Effect of strain localization on frictional behavior of sheared granular materials. Journal of Geophysical Research, 115, B01204, 2010.

[14] Cabalar, A.F. The effects of fines on the behaviour of a sand mixture. Geotech. Geol. Eng., 29, 91-100, 2011.

[15] Cabalar, A.F., Dulundu, K., Tuncay, K. Strength of various sands in triaxial and cyclic direct shear tests. Engineering Geology, 156, 92-102, 2013.

[16] Benahmed, N., Nguyen, T.K., Hicher, P.Y., Nicolas, M. An experimental investigation into the effects of low plastic fines content on the behaviour of sand/silt mixtures. European Journal of Environmental and Civil Engineering, 19 (1), 109-128, 2015.

[17] Brace, W.F., Byerlee, J.D. Stick-slip as a mechanism for earthquakes, Science, 153, 990-992, 1966.

[18] Aharonov, E., Sparks, D. Stick-slip motion in simulated granular layers. J. Geophys. Res., 109, B09306, 2004.

[19] Savage, H.M., Marone, C. Effects of shear velocity oscillations on stick-slip behavior in laboratory experiments. J. Geophys. Res., 112, B02301, 2007.

[20] Alshibli, K.A., Roussel, L.E. Experimental investigation of slip-stick behaviour in granular materials. Int. J. Numer. Anal. Meth. Geomech., 30, 1391-1407, 2006.

[21] Thompson, P.A. and Grest, G.S. (1991). Granular flow: Friction and Dilatancy Transition. Physical Review Letters, Vol. 67, No. 13, 1751- 1754.

[22] Feder, H.J., and Feder, J. (1991). Self-organized critically in a stick-slip process. Physical Review Letters, Vol. 66, No. 20, 2669-2672.

[23] Demirel, A.L. and Granick, S. (1996). Friction fluctuations and friction memory in stick-slip motion. Physical Review Letters, Vol. 77, No. 21, 4330- 4333. 
[24] Miller, B., O’Hern, C., and Behringer, R.P. (1996). Stress fluctuations for continuously sheared granular materials. Physical Review Letters, Vol. 77, No. 15, 3110- 3113.

[25] Nasuno, S., Kudrolli, A., Bak, A., and Gollub, J.P. (1998). Time-resolved studies of stick-slip friction in sheared granular layers. Physical Review E, Vol. 58, No. 2. 21612171.

[26] Albert, I., Tegzes, P., Kahng, B., Albert, R., Sample, J.G., and Pfeifer, M. (2000). Jamming and fluctuations in granular drag. Physical Review Letters, Vol. 84, No. 22, 5122-5125.

[27] Cain, R.G., Page, N.W., and Biggs, S. (2001). Microscopic and macroscopic aspects of stick-slip motion in granular shear. Physical Review E, Vol. 64, 016413.

[28] Gourdon, D. and Israelachvili, J.N. (2003). Transitions between smooth and complex stick-slip sliding of surfaces. Physical Review E, Vol. 68, No. 021602.

[29] Kim, M.S. Etude expérimentale du comportement mécanique des matériaux granulaires sous forte contrainte, Thèse de doctorat, Ecole Centrale Paris, 1995.

[30] Duchesne, A. Etude du comportement mécanique d'un combustible granulaire soumis a des sollicitations d'origine thermique dans un propulseur thermonucléaire spatial. Thèse de doctorat, Ecole Centrale Paris, 1998.

[31] Adjemian, F. Stick-slip et transition de broutage dans les essais triaxiaux sur billes de verre. Thèse de doctorat, Ecole Centrale Paris, 2003.

[32] Adjemian, F., Evesque, P. Experimental study of stick-slip behaviour. Int. J. Numer. Anal. Meth. Geomech., 28, 501-530, 2004.

[33] Roussel, L.E. Experimental investigation of stick-slip behavior in granular materials. Master thesis, Louisiana State University, 2005.

[34] Cabalar, A.F., Clayton, C.R.I. Some observations of the effects of pore fluids on the triaxial behaviour of a sand. Granular Matter, 12, 87-95, 2010.

[35] Doanh, T., Hoang, M.T., Roux, J.-N., Dequeker, C. Stick-slip behaviour of model granular materials in drained triaxial compression. Granular Matter, 15, 1-23, 2013.

[36] Ozbay, A., Cabalar, A.F. Effects of triaxial confining pressure and strain rate on stickslip behavior of a dry granular material. Granular Matter, 18 (3), 60, 2016.

[37] Gajo, A. (2004). The influence of system compliance on collapse of triaxial sand samples. Canadian Geotechnical Journal, Vol. 41, 257- 273.

[38] Luding, S. (2003). The micro-macro mechanics of granular materials. GACM report 2, $22-28$. 